\title{
The Need for Effective Adjuvant Therapy in Uterine Leiomyosarcoma: A Single-centre Experience
}

\author{
Emrah Eraslan, Fatih Yildiz, Aysegul Ilhan and Mutlu Dogan \\ Department of Medical Oncology, Dr. Abdurrahman Yurtaslan Ankara Oncology Training and Research Hospital, Ankara, Turkey
}

\begin{abstract}
Objective: To evaluate the efficacy of adjuvant chemotherapy (ACTX) in completely resected uterine leiomyosarcoma (ULMS) in terms of survival outcomes.

Study Design: Descriptive study.

Place and Duration of Study: Department of Medical Oncology, Dr. Abdurrahman Yurtaslan Ankara Oncology Training and Research Hospital, Ankara, Turkey from February 2009 to November 2019.

Methodology: Patients older than 18 years, who underwent complete surgical resection with a diagnosis of non-metastatic ULMS were evaluated retrospectively. The patients were divided into two groups: patients who received ACTx (group I) and patients who received only surgical treatment (group II). Both groups were compared in terms of main patient and tumour characteristics, relapse rates, relapse-free survival (RFS) and overall survival (OS).

Results: Forty-five patients with a median age of 52.1 years (IQR, 45.8-58.2) were included in the study. Group I consisted of $26(57.8 \%)$ patients and group II consisted of $19(42.2 \%)$ patients. Median RFS was 43.8 months $(95 \% \mathrm{Cl}, 7.4-80.2)$ and the median OS was 81.3 months $(95 \% \mathrm{Cl}, 39.4-123.1)$ for all patients $(\mathrm{N}=45)$. Median RFS was $27.1 \mathrm{months}(95 \% \mathrm{Cl}, 6.8-47.4)$ in group I $(\mathrm{n}=26)$ and 43.8 months $(95 \% \mathrm{Cl}, 11.8-75.8)$ in group II $(\mathrm{n}=19)(p=0.985)$. Median OS was 85.6 months $(95 \% \mathrm{Cl}$, 38.3-132.9) in group I $(n=26)$ and 81.2 months $(95 \% \mathrm{Cl}, 62.1-100.4)$ in group II $(n=19)(p=0.699)$.

Conclusion: There was no survival benefit of ACTx in completely resected ULMSs, in accordance with the literature data. There is a need for prospective randomised clinical trials evaluating the role of ACTx in ULMSs.
\end{abstract}

Key Words: Uterine leiomyosarcoma, Complete resection, Adjuvant chemotherapy, Relapse, RFS, OS.

How to cite this article: Eraslan E, Yildiz F, Ilhan A, Dogan M. The Need for Effective Adjuvant Therapy in Uterine Leiomyosarcoma: A Single-centre Experience. J Coll Physicians Surg Pak 2021; 31(08):926-931.

\section{INTRODUCTION}

Uterine leiomyosarcoma (ULMS), a rare gynaecological malignancy, accounts for $1 \%$ of all uterine malignancies; whereas, $70 \%$ of all uterine sarcomas are ULMSs. ${ }^{1,2}$ It is most common between the ages of 40-69 years, and the average age at diagnosis is 51 years. ${ }^{3,4}$ The primary treatment modality for early-stage ULMSs is hysterectomy and complete surgical resection of the gross tumour. ${ }^{4}$ The recurrence rate has been reported as 50 $-70 \%$, even in those who had radical surgical treatment. ${ }^{5}$ Uterine leiomyosarcoma has an aggressive clinical course with a 5 -year progression-free survival (PFS) rate of $30 \%$ and 5 -year overall survival (OS) rates as $76 \%, 60 \%$, and $45 \%$ in stage I, II, III surgically resected ULMS, respectively. ${ }^{6,7}$

Correspondence to: Dr. Emrah Eraslan, Department of Medical Oncology, Dr. Abdurrahman Yurtaslan Ankara Oncology Training and Research Hospital, Ankara, Turkey E-mail: dremraheraslan@gmail.com

Received: March 21, 2021; Revised: May 01, 2021;

Accepted: June 30, 2021

DOI: https://doi.org/10.29271/jcpsp.2021.08.926
It has been reported that tumour stage, tumour size, mitotic count, and percentage of necrosis have prognostic significance in ULMS. ${ }^{4}$ Though grade is a prognostic factor in many solid tumours, its prognostic significance has not been well established in ULMS.

The presence of a very aggressive clinical course despite surgical treatment, even in uterine confined disease, clearly indicates the need for adjuvant therapy. The use of adjuvant chemotherapy (ACTX) has increased in the treatment of ULMS in recent years. ${ }^{8}$ However, it is controversial that ACTx reduces relapse or death rates. ${ }^{9}$ Moreover, since it is a rare disease, it is difficult to conduct randomised prospective trials on the efficacy of ACTx in the treatment of ULMS, and the literature data is mostly based on retrospective studies or case series. ${ }^{9}$

The aim of this study was to evaluate the efficacy of ACTx in completely resected ULMS in terms of survival outcomes, and also to evaluate the prognostic significance of clinicopathologicalcharacteristics.

\section{METHODOLOGY}

The patients followed up with a diagnosis of radically surgically resected non-metastatic ULMS between February 2009 and November 2019 at the Medical Oncology Clinic of Dr. Abdur- 
rahman Yurtaslan Ankara Oncology Training and Research Hospital, Ankara, Turkey, were included in this retrospective study. The patients with sufficient data in medical records were evaluated retrospectively after the Ethical Committee approval. The patients who had distant metastasis at diagnosis, severe comorbidities (such as uncontrolled cardiovascular diseases, uncontrolled cerebrovascular diseases, renal failure requiring dialysis), incomplete/suboptimal surgical resection and who could not have completed the planned ACTx, were excluded from the study. Adjuvant chemotherapy regimens were as gemcitabine/docetaxel regimen [gemcitabine $\left(1 \mathrm{~g} / \mathrm{m}^{2} /\right.$ day, $\mathrm{d} 1$ and $\mathrm{d} 8$, every 21 days) and docetaxel $\left(75 \mathrm{mg} / \mathrm{m}^{2}, \mathrm{~d} 1\right.$, every 21 days) and IMA regimen [doxorubicin $\left(60 \mathrm{mg} / \mathrm{m}^{2}, \mathrm{dl}\right.$, every 21 days) and ifosfamide ( $3 \mathrm{~g} / \mathrm{m}^{2} /$ day, d1-d3, and Mesna 3 $\mathrm{g} / \mathrm{m}^{2} /$ day, d1-d3, every 21 days)].

Age at diagnosis, tumour characteristics (size, mitotic count, necrosis rate, Ki 67 index), stage, adjuvant chemotherapy regimens with the number of cycles were recorded. Patients with surgically treated ULMS were divided into two groups according to their status of receiving ACTx. Group I was formed from patients who received ACTX, and group II consisted of patients treated with only surgery. Groups were compared forage, menopausal status (premenopausal or postmenopausal), stage (stage I or stage II-IVA), tumour characteristics (tumour size [mm], mitotic count [per 10 high-power fields (HPF)], necrosis ratio [\%], Ki 67 proliferation index [\%]). They were also compared in terms of recurrence rate, relapse-free survival (RFS), and OS. In addition, the patients were subgrouped according to menopausal status (premenopausal versus postmenopausal), stage (stage I versus stage II-IVA), tumour size (< median versus $\geq$ median), mitotic count $(<20$ versus $\geq 20$, per $10 \mathrm{HPF}$ ), necrosis rate ( $<50 \%$ versus $\geq 50 \%$ ) Ki 67 proliferation index ( $<$ median versus $\geq$ median). These subgroups were compared forRFS and OS.

Descriptive statistics were used to show the distribution of the main characteristics of the population. The groups' differences in categorical and ordinal parameters were evaluated by using Chi-square and Mann-Whitney U-tests, respectively. Relapsefree survival was defined as the time from radical surgical intervention to the date of relapse or death. Overall survival was defined as the time from diagnosis to death or last follow-up. Survival rates were estimated using the Kaplan-Meier method, and the groups were compared using the log-rank test for differences in survival. Statistical analysis was performed using SPSS software (SPSS for Windows, version 24.0, SPSS Inc., Chicago, USA). All statistical tests were two-sided, and a $p$-value $(<0.05)$ was considered statistically significant.

This retrospective study was conducted after the local Ethics Committee's approval (No. 2021-01/942, Date:13.01.2021).

\section{RESULTS}

Forty-five patients with a median age of 52.1 years (IQR, 45.8-58.2) were included in the study. Median follow-up was
20.8 months (IQR, 9.1-42.1). Fourteen (31.1\%) patients were premenopausal, while 31 (68.9\%) were postmenopausal. Thirty-one $(68.9 \%), 1(2.2 \%), 8(17.8 \%)$, and $5(11.1 \%)$ patients had stage I, II, III and IVA disease, respectively. Considering tumour characteristics, median tumour size was $80 \mathrm{~mm}$ (IQR, 55.0-120.0), median mitotic count was 25 / per $10 \mathrm{HPF}$ (IQR, 11.5-31.0), median necrosis rate was $27.5 \%$ (IQR, 10.0-52.5), median value of the Ki 67 index was $30.0 \%$ (IQR, 20.0-50.0). Group I consisted of 26 (57.8\%) patients and group II consisted of $19(42.2 \%)$ patients. Of the patients receiving ACTx, 20 (76.9\%) patients had four cycles of gemcitabine/docetaxel [gemcitabine $\left(1 \mathrm{~g} / \mathrm{m}^{2} /\right.$ day, $\mathrm{d} 1$ and $\mathrm{d} 8$, every 21 days) and docetaxel $\left(75 \mathrm{mg} / \mathrm{m}^{2}, \mathrm{~d} 1\right.$, every 21 days)], and $6(23.1 \%)$ patients had four cycles of IMA regimen [doxorubicin $\left(60 \mathrm{mg} / \mathrm{m}^{2}, \mathrm{~d} 1\right.$, every 21 days) and ifosfamide $\left(3 \mathrm{~g} / \mathrm{m}^{2} / \mathrm{day}, \mathrm{d} 1-\mathrm{d} 3\right.$, and Mesna 3 $\mathrm{g} / \mathrm{m}^{2} /$ day, d1-d3, every 21 days)]. Baseline patients' and tumour characteristics are displayed in Tablel.

Relapse occurred in 27 (60\%) patients. Of the patients with relapse, 5 (18.5\%) had local recurrence, and 22 (81.5\%) had recurrence with distant organ metastasis. Distant organ metastasis sites were lung, bone, peritoneum, liver, and lymph node for $20(74.1 \%), 5(18.5 \%), 4(14.8 \%), 3(11.1 \%)$, and $1(3.7 \%)$ patients, respectively. Six (33.3\%) of 18 patients without relapse and $13(48.1 \%)$ of 27 patients with relapse had received $\operatorname{ACTx}(p=0.324)$. When the effect of age, menopausal status, tumour characteristics (tumour size, mitotic count, necrosis ratio, Ki 67 proliferation index) on recurrence was evaluated, there was no statistically significant difference between those with and without recurrence for any of these factors (Table II).

Median RFS was 43.8 months ( $95 \% \mathrm{Cl}, 7.4-80.2)$ (Figure $1 \mathrm{~A}$ ) and the median OS was 81.3 months $(95 \% \mathrm{Cl}, 39.4-123.1)$ (Figure $1 C)$ for all patients $(\mathrm{N}=45)$. There was no difference for RFS and OS between group I and group II ( $p=0.985, p=0.699)$. Median RFS was 27.1 months $(95 \% \mathrm{Cl}, 6.8-47.4)$ in group I $(n=26)$ and 43.8 months $(95 \% \mathrm{Cl}, 11.8-75.8)$ in group II $(\mathrm{n}=19)$ (Figure 1B) $(p=0.985)$. Median OS was 85.6 months $(95 \% \mathrm{Cl}, 38.3-132.9)$ in group I $(n=26)$ and 81.2 months $(95 \% \mathrm{Cl}, 62.1-100.4)$ in group II $(\mathrm{n}=19$, Figure 1D, $p=0.699$ ).

The survival analysis results performed by grouping the patients according to their menopausal status, stage at diagnosis and tumour characteristics (tumour size, mitotic count, necrosis ratio, Ki 67 proliferation index) are shown in Table III.

\section{DISCUSSION}

In this study, ACTx affects relapse rate and survival in surgically resected ULMS were investigated. It was observed that ACTX had not provided any benefit for RFS or OS. A recent meta-analysis by Rizzo et al. including nine studies, in which 252 patients had ACTx and 293 patients had been followed up after surgery, and it was observed that the recurrence rate did not decrease with ACTx. ${ }^{9}$ Similarly, in the retrospective study of Mancari et al., which included 140 patients with operated ULMS, 5-year OS rates were reported as $68.7 \%$ and $65.6 \%$ for the patients who received ACTx and those who did not, respectively $(p=0.521){ }^{10}$ 


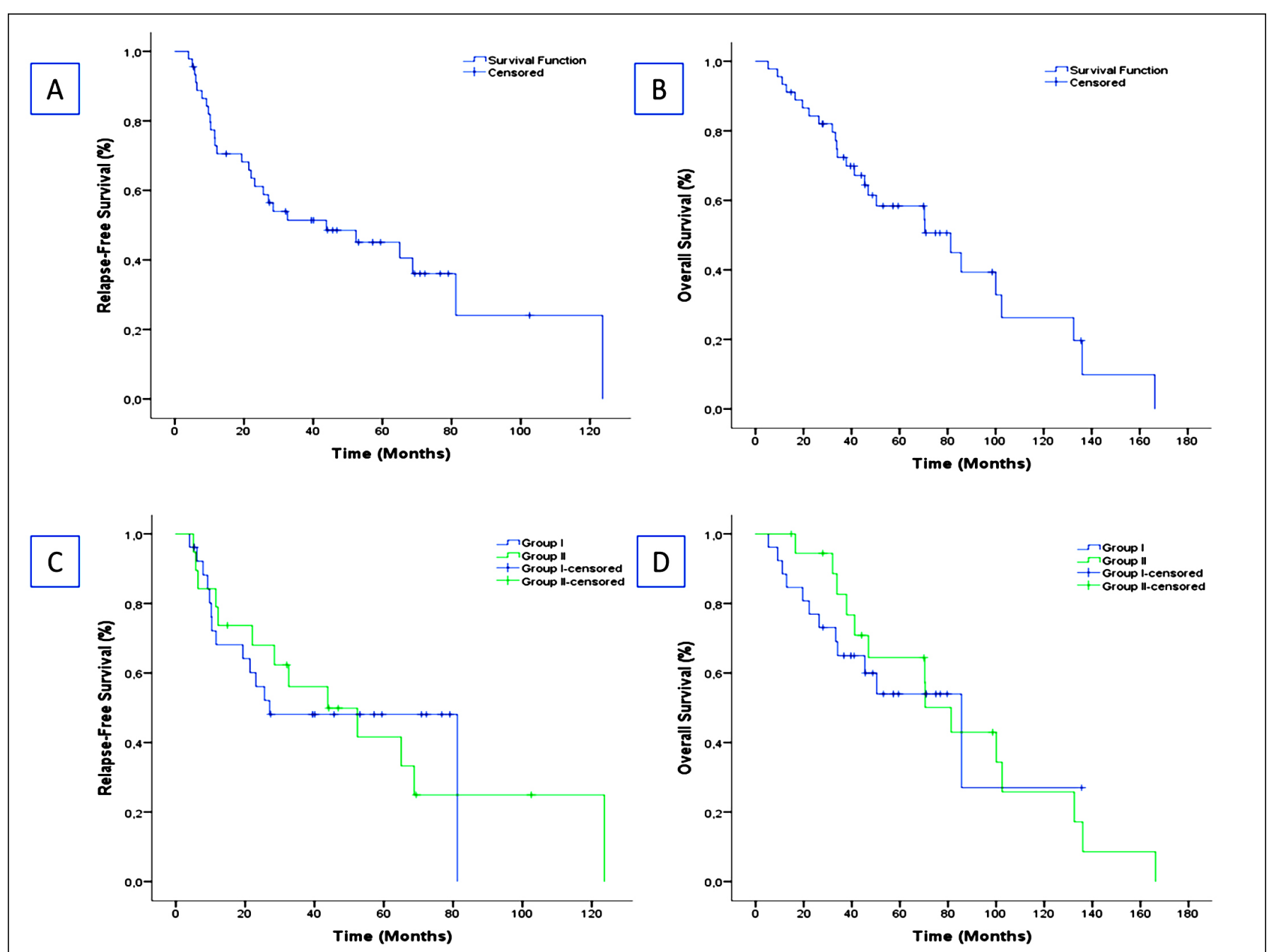

Figure 1: Kaplan-Meier survival curves: RFS (A) and OS (B) for all patients (N = 45); RFS (C) and OS (D) for group I ( $n=26)$ and group II ( $n=19$ ).

Data is limited for the benefit of ACTX in ULMS. ${ }^{5,11-13}$ However, these studies were generally designed to include all soft-tissue sarcomas, and ULMSs were included as a subgroup.

There are conflicting results regarding the benefit of ACTx for all soft-tissue sarcomas. Studies that failed to show the survival benefit of ACTx in soft-tissue sarcomas are mostly early monotherapy studies. ${ }^{13}$ However, more recent, a prospective study in which patient recruitment was stopped early due to the early demonstration of a clear DFS benefit by the Italian study group showed a survival benefit with adjuvant doxorubicin $\left(60 \mathrm{mg} / \mathrm{m}^{2}\right) /$ ifosfamide $\left(9 \mathrm{~g} / \mathrm{m}^{2}\right)$ combination in operated high-risk soft-tissue sarcomas. ${ }^{14}$ Similarly a meta-analysis by Pervaiz et al. reported a significant decrease in mortality with ifosfamide/doxorubicin combination ACTx for soft-tissue sarcomas ( $\mathrm{HR}=0.56,95 \% \mathrm{Cl}$, $0.36-0.85 ; p=0.01) .{ }^{15}$ Uterine leiomyosarcomas may have a different clinical course when compared to other soft-tissue sarcomas. Mancari et al. failed to show a survival benefit of ACTx, but the vast majority of the patients (54 of 64 patients) received ifosfamide/doxorubicin combination therapy as ACTx, and lack of survival benefit might have been related to the ACTx regimen. ${ }^{10}$ Gemcitabine/docetaxel combination regimen was associated with a $35.8 \%$ objective response rate and $26.2 \%$ stable disease rate, when it was given as first-line therapy in a phase II study involving 42 patients diagnosed with advanced ULMS. ${ }^{16} \mathrm{~A}$ prospective study in which Hensley et al. included 25 patients diagnosed with completely resected stages I-IV high-grade ULMS, 2-year PFS rate with adjuvant gemcitabine/docetaxel treatment was superior to historical control rates. ${ }^{17}$ A phase III trial of four cycles of gemcitabine/docetaxel combination chemotherapy followed by four cycles of doxorubicin versus observation in uterus confined high-grade LMS was prematurely closed due to slow accrual. ${ }^{18}$ Hence, it failed to clear the survival benefit of ACTx in ULMS. In the literature, retrospective studies evaluating the effectiveness of the gemcitabine/docetaxel combination regimen in the adjuvant setting did not show that ACTX provides any RFS or OS benefit. ${ }^{8,10,19-21}$ In this study, $76.9 \%$ of the patients in the ACTx group received a gemcitabine/docetaxel combination regimen, and the authors could not have observed any survival benefit. Unfortunately, this study is compatible with the literature data regarding the inefficacy of ACTX in ULMS. 


Table I: Baseline patients' and tumour characteristics.
\begin{tabular}{|l|l|l|l|l|}
\hline Characteristic & All patients (N = 45) & Group I (n = 26) & Group II (n = 19) & p-value \\
\hline Age at diagnosis (Median, IQR) & $52.1(45.8-58.2)$ & $50.1(47.5-54.7)$ & $53.3(45.5-59.9)$ \\
\hline Menopausal status, n (\%) & $14(31.1 \%)$ & $10(22.2 \%)$ & $4(8.9 \%)$ \\
\hline Premenopausal & $31(68.9 \%)$ & $16(35.6 \%)$ & $15(33.3 \%)$ \\
\hline Postmenopausal & $31(68.9 \%)$ & $14(31.1 \%)$ & $17(37.8 \%)$ \\
\hline Stage at diagnosis, n (\%) & $12(26.7 \%)$ & $2(4.4 \%)$ \\
\hline Stage I & $14(31.1 \%)$ & $113.0(60.0-132.5)$ & $60.0(50.0-87.5)$ \\
\hline Stage II-IVA & $80.0(55.0-120.0)$ & $15.0(10.5-27.5)$ & 0.213 \\
\hline Tumour size, mm (Median, IQR) & $25.0(11.5-31.0)$ & $30.0(16.25-32.75)$ & $\mathbf{0 . 0 1 1}$ \\
\hline Mitotic count, (per 10 HPF) (Median, IQR) & $27.5(10.0-52.5)$ & $45.0(10.0-60.0)$ & $10.0(3.75-50.0)$ \\
\hline Necrosis ratio, \% (Median, IQR) & $30.0(20.0-50.0)$ & $\mathbf{0 . 0 0 8}$ & 0.103 \\
\hline Ki 67 index, \% (Median, IQR) & $40.0(22.5-55.0)$ & $25.0(20.0-47.5)$ \\
\hline ACTx: Adjuvant chemotherapy; IQR: Interquartile of range; HPF: High-power fields. & 0.065 \\
\hline
\end{tabular}

\begin{tabular}{|c|c|c|c|}
\hline \multirow[b]{2}{*}{ Characteristic } & \multicolumn{2}{|c|}{ Relapse } & \multirow[b]{2}{*}{ p-value } \\
\hline & $\begin{array}{l}\text { No } \\
n=18,(\% 40)\end{array}$ & $\begin{array}{l}\text { Yes } \\
n=27(\% 60)\end{array}$ & \\
\hline Age at diagnosis (Median, IQR) & $53.4(48.6-60.5)$ & $51.5(42.4-55.7)$ & 0.172 \\
\hline \multicolumn{3}{|l|}{ Menopausal status, $\mathrm{n}(\%)$} & 0.293 \\
\hline Premenopausal & $4(\% 22.2)$ & $10(\% 37.0)$ & \\
\hline Postmenopausal & $14(\% 77.8)$ & $17(\% 63.0)$ & \\
\hline \multicolumn{3}{|l|}{ Stage at diagnosis } & 0.793 \\
\hline Stage I & $12(\% 66.7)$ & $19(\% 70.4)$ & \\
\hline Stage II-IVA & $6(\% 33.3)$ & $8(\% 29.6)$ & \\
\hline Tumour size, mm (Median, IQR) & $90.0(57.5-125.0)$ & $75.0(53.75-110.0)$ & 0.681 \\
\hline Mitotic count (Median, IQR) & $22.0(11.0-30.0)$ & $25.0(14.25-34.25)$ & 0.497 \\
\hline Necrosis ratio, \% (Median, IQR) & $20.0(10.0-57.5)$ & $32.5(5.0-52.5)$ & 0.794 \\
\hline Ki 67 index (Median, IQR) & $25.0(20.0-40.0)$ & $40.0(20.0-52.5)$ & 0.297 \\
\hline
\end{tabular}

Table III: Survival analysis for subgroups.

\begin{tabular}{|c|c|c|c|c|}
\hline Parameter & $\begin{array}{l}\text { RFS } \\
\text { Median (months) } \\
\text { (95\% Cl, range) }\end{array}$ & p-value & $\begin{array}{l}\text { OS } \\
\text { Median (months) } \\
\text { (95\% Cl, range) }\end{array}$ & p-value \\
\hline All patients $(\mathrm{N}=45)$ & $43.8(7.4-80.2)$ & & $81.3(39.4-123.1)$ & \\
\hline \multicolumn{2}{|l|}{ Menopausal status } & 0.826 & & 0.382 \\
\hline Premenopausal $(n=14)$ & $25.6(6.7-44.5)$ & & $37.9(9.8-66.0)$ & \\
\hline Postmenopausal $(n=31)$ & $52.4(9.3-95.5)$ & & $81.2(59.7-102.8)$ & \\
\hline \multicolumn{2}{|l|}{ Stage at diagnosis } & 0.293 & & 0.110 \\
\hline Stage I $(n=31)$ & $52.4(7.8-96.9)$ & & $81.2(48.8-113.7)$ & \\
\hline Stage II-IVA $(n=14)$ & $19.4(1.3-37.4)$ & & 85.6 & \\
\hline \multicolumn{2}{|l|}{ Tumour size } & 0.341 & & 0.465 \\
\hline$<$ Median ( $n=19)$ & 68.7 (29.7-107.8) & & $81.2(47.5-115.0)$ & \\
\hline$\geq$ Median $(n=20)$ & $43.8(12.2-75.4)$ & & $70.4(19.8-121.1)$ & \\
\hline \multicolumn{2}{|l|}{ Mitotic count } & 0.425 & & 0.922 \\
\hline$<20(\mathrm{n}=11)$ & 68.7 & & 100.1 & \\
\hline$\geq 20(n=18)$ & $81.2(1.4-161.1)$ & & $102.6(2.7-202.5)$ & \\
\hline \multicolumn{2}{|l|}{ Necrosis ratio } & 0.344 & & 0.935 \\
\hline$<\% 50(n=15)$ & $65.1(28.1-102.0)$ & & $100.1(35.7-164.5)$ & \\
\hline$\geq \% 50(n=11)$ & $23.1(0.0-56.5)$ & & $50.3(38.7-62.0)$ & \\
\hline Ki 67 index & & 0.212 & & 0.574 \\
\hline$<$ Median $(n=12)$ & $65.1(43.0-87.1)$ & & $81.3(64.8-97.7)$ & \\
\hline$\geq$ Median $(n=11)$ & $10.3(0.0-28.7)$ & & $132.5(4.5-260.5)$ & \\
\hline \multicolumn{2}{|l|}{ Adjuvant chemotherapy } & 0.985 & & 0.699 \\
\hline Group I $(n=26)$ & $27.1(6.8-47.4)$ & & $85.6(38.3-132.9)$ & \\
\hline Group II (n = 19) & $43.8(11.8-75.8)$ & & $81.2(62.1-100.4)$ & \\
\hline
\end{tabular}

Since this study is a retrospective study, there might have been a patient selection bias. When the group I and group II were compared, patients in group I were more likely to have an advanced stage (i.e., stage II-IVA), and median tumour size was larger than in group II. Although not statistically significant, the mitotic count, necrosis ratio, and Ki 67 proliferative index ratio for the group I patients were also numerically higher than those in group II. From this point of view, the ACTx group patients seem to have a higher risk of relapse. On the other hand, the patients who have less risk for relapse might have had a better clinical course even if they had not received ACTx, which might have contributed to the inefficacy of ACTx in these patients. However, when the authors compared the groups in terms of age, menopausal status, stage and tumour characteristics (tumour size, mitotic count, necrosis ratio, and Ki 67 proliferative index), there was no difference for relapse between these two groups. Similarly, there was no significant difference in RFS or OS. It is 
known that tumour stage, tumour size, mitotic count, and percentage of necrosis have prognostic significance in ULMS. ${ }^{4}$ Although Kim et al. could not show any benefit of ACTx in ULMS, they found that low mitotic count was associated with a better progression-free interval in early-stage disease. ${ }^{19}$ Mitotic index, age, and tumour size appear as significant parameters in a prognostic nomogram developed for ULMS by Memorial Sloan-Kettering Cancer Center. ${ }^{22,23}$ There is a need for well-designed prospective randomised trials evaluating the effectiveness of ACTx in ULMS, in which patients are well balanced in terms of stage, age, menopausal status and tumour characteristics. MSKCC's nomogram can also be used as a tool for patient selection for these studies.

Determination of molecular pathophysiological mechanisms in ULMS might contribute to the development of novel agents such as targeted therapies. There is a wide gap in the literature data in this area as well.

\section{CONCLUSION}

Uterine leiomyosarcomas have a relatively poor prognosis even, if completely resected early-stage tumours confined to the uterus. There is no well documented current adjuvant treatment modality with absolute survival benefit in surgically resected ULMS. The present study showed no survival benefit of ACTx in completely resected ULMSs. In conclusion, the authors need prospective randomised clinical trials evaluating the role of ACTx in ULMSs.

\section{ETHICAL APPROVAL:}

The medical records of these patients were reviewed retrospectively after obtaining the approval of the local Ethics Committee (UHS Dr. Abdurrahman Yurtaslan Ankara Oncology Training and Research Hospital Ethics Committee, No. 2021-01/942, Date: 13.01.2021).

\section{PATIENTS' CONSENT:}

All patients or their legal representatives signed an informed consent document.

\section{CONFLICT OF INTEREST:}

The authors declared no conflict of interest.

\section{AUTHORS' CONTRIBUTION:}

EE: Conception and design, analyses and interpretaion of data, drafting of manuscript.

FY: Conception and design, analyses and interpretation of data.

Al: Acquisition of data.

MD: Conception and design, reviewing the paper and final approval.

\section{REFERENCES}

1. Society AC. Cancer facts \& figures 2018. Atlanta, GA2018.

2. Ricci S, Stone RL, Fader AN. Uterine leiomyosarcoma: Epidemiology, contemporary treatment strategies and the impact of uterine morcellation. Gynecol Oncol 2017;
145(1):208-16. doi: 10.1016/j.ygyno.2017.02.019.

3. Mbatani N, Olawaiye AB, Prat J. Uterine sarcomas. Int J Gynaecol Obstet 2018; 143 Suppl 2:51-8. doi: 10.1002/ijgo. 12613.

4. Ganjoo KN. Uterine sarcomas. Curr Probl Cancer 2019; 43(4):283-8. doi: 10.1016/j.currproblcancer.2019.06.001.

5. Giuntoli RL, 2nd, Metzinger DS, DiMarco CS, Cha SS, Sloan JA, Keeney GL, et al. Retrospective review of 208 patients with leiomyosarcoma of the uterus: Prognostic indicators, surgical management, and adjuvant therapy. Gynecol Oncol 2003; 89(3):460-9. doi: 10.1016/s0090-8258(03) 00137-9.

6. Zivanovic O, Leitao MM, lasonos A, Jacks LM, Zhou Q, AbuRustum NR, et al. Stage-specific outcomes of patients with uterine leiomyosarcoma: A comparison of the international Federation of gynecology and obstetrics and american joint committee on cancer staging systems. J Clin Oncol 2009; 27(12):2066-72. doi: 10.1200/JCO.2008.19.8366.

7. Hensley ML, Barrette BA, Baumann K, Gaffney D, Hamilton $\mathrm{AL}$, Kim JW, et al. Gynecologic cancer InterGroup (GCIG) consensus review: Uterine and ovarian leiomyosarcomas. Int J Gynecol Cancer 2014; 24(9 Suppl 3):S61-6. doi: 10.1097/ IGC.0000000000000261.

8. Littell RD, Tucker LY, Raine-Bennett T, Palen TE, Zaritsky E, Neugebauer $\mathrm{R}$, et al. Adjuvant gemcitabine-docetaxel chemotherapy for stage I uterine leiomyosarcoma: Trends and survival outcomes. Gynecol Oncol 2017; 147(1):11-7. doi: 10.1016/j.ygyno.2017.07.122.

9. Rizzo A, Nannini M, Astolfi A, Indio V, De laco P, Perrone AM, et al. Impact of chemotherapy in the adjuvant setting of early stage uterine leiomyosarcoma: A systematic review and updated meta-analysis. Cancers (Basel) 2020; 12(7): 1899. doi: 10.3390/cancers12071899.

10. Mancari R, Signorelli M, Gadducci A, Carinelli S, De Ponti E, Sesana S, et al. Adjuvant chemotherapy in stage I-II uterine leiomyosarcoma: A multicentric retrospective study of 140 patients. Gynecol Oncol 2014; 133(3):531-6. doi: 10.1016/ j.ygyno.2014.03.001.

11. Bramwell VH. Adjuvant chemotherapy for adult soft tissue sarcoma: Is there a standard of care? J Am Soc Clin Oncol 2001; 19(5):1235-7. doi: 10.1200/JCO.2001.19.5.1235.

12. Woll PJ, Reichardt P, Le Cesne A, Bonvalot S, Azzarelli A, Hoekstra $\mathrm{HJ}$, et al. Adjuvant chemotherapy with doxorubicin, ifosfamide, and lenograstim for resected soft-tissue sarcoma (EORTC 62931): A multicentre randomised controlled trial. Lancet Oncol 2012; 13(10):1045-54. doi: 10.1016/S1470-2045(12)70346-7.

13. Tierney JF, Mosseri V, Stewart LA, Souhami RL, Parmar MK. Adjuvant chemotherapy for soft-tissue sarcoma: Review and meta-analysis of the published results of randomised clinical trials. Br J Cancer 1995; 72(2):469-75. doi: 10.1038/ bjc.1995.357.

14. Frustaci S, Gherlinzoni F, De Paoli A, Bonetti M, Azzarelli A, Comandone $A$, et al. Adjuvant chemotherapy for adult soft tissue sarcomas of the extremities and girdles: Results of the Italian randomised cooperative trial. J Clinical Oncol 2001; 19(5):1238-47. doi: 10.1200/JCO.2001.19.5.1238.

15. Pervaiz N, Colterjohn N, Farrokhyar F, Tozer R, Figueredo A, Ghert M. A systematic meta-analysis of randomised controlled trials of adjuvant chemotherapy for localised 
resectable soft-tissue sarcoma. Cancer 2008; 113(3): 573-81. doi: 10.1002/cncr.23592.

16. Hensley ML, Blessing JA, Mannel R, Rose PG. Fixed-dose rate gemcitabine plus docetaxel as first-line therapy for metastatic uterine leiomyosarcoma: A gynecologic oncology group phase II trial. Gynecol Oncol 2008; 109(3):329-34. doi: 10.1016/j.ygyno.2008.03.010.

17. Hensley ML, Ishill N, Soslow R, Larkin J, Abu-Rustum N, Sabbatini $\mathrm{P}$, et al. Adjuvant gemcitabine plus docetaxel for completely resected stages I-IV high grade uterine leiomyosarcoma: Results of a prospective study. Gynecol Oncol 2009; 112(3):563-7. doi: 10.1016/j.ygyno.2008. 11.027.

18. Hensley ML, Enserro D, Hatcher H, Ottevanger PB, KrarupHansen A, Blay JY, et al. Adjuvant gemcitabine plus docetaxel followed by doxorubicin versus observation for high-grade uterine leiomyosarcoma: A phase III NRG oncology/gynecologic oncology group study. J Clin Oncol 2018; 36(33):Jco1800454. doi: 10.1200/JCO.18.00454.

19. Kim WY, Chang SJ, Chang KH, Yoon JH, Kim JH, Kim BG, et al. Uterine leiomyosarcoma: 14-year two-center experience of
31 cases. Cancer Res Treat 2009; 41(1):24-8. doi: 10.4143/crt.2009.41.1.24.

20. Ricci S, Giuntoli RL, 2nd, Eisenhauer E, Lopez MA, Krill L, Tanner EJ, 3rd, et al. Does adjuvant chemotherapy improve survival for women with early-stage uterine leiomyosarcoma? Gynecol Oncol 2013; 131(3):629-33. doi: 10.1016/j.ygyno.2013.08.037.

21. Kim SI, Choi CH, Kim K, Hong DH, Park JY, Kwon BS, et al. Effectiveness of adjuvant treatment for morcellated, International federation of gynecology and obstetrics stage I uterine leiomyosarcoma: A korean multicenter study. J Obstet Gynaecol Res 2020; 46(2):337-46. doi: 10.1111/ jog. 14171.

22. Zivanovic O, Jacks LM, lasonos A, Leitao MM, Jr, Soslow RA, Veras $\mathrm{E}$, et al. A nomogram to predict postresection 5-year overall survival for patients with uterine leiomyosarcoma. Cancer 2012; 118(3):660-9. doi: 10.1002/cncr.26333.

23. Iasonos A, Keung EZ, Zivanovic O, Mancari R, Peiretti M, Nucci $M$, et al. External validation of a prognostic nomogram for overall survival in women with uterine leiomyosarcoma. Cancer 2013; 119(10):1816-22. doi: 10.1002/cncr.27971. 